Acta Crystallographica Section B

Structural

Science

ISSN 0108-7681

Sarah A. Barnett, ${ }^{\mathrm{a} *}$ Charlotte K. Broder, ${ }^{\mathbf{b}}$ Kenneth Shankland, ${ }^{\mathrm{b}}$ William I. F. David, ${ }^{\text {b }}$ Richard M. Ibberson $^{b}$ and Derek A. Tocher ${ }^{a}$

aDepartment of Chemistry, University College London, 20 Gordon Street, London WC1H OAJ, England, and ${ }^{\mathbf{b}}$ ISIS Facility, CCLRC Rutherford Appleton Laboratory, Chilton, Didcot OX11 OQX, England

Correspondence e-mail: sarah.barnett@ucl.ac.uk

\section{Single-crystal X-ray and neutron powder diffraction investigation of the phase transition in tetrachloro- benzene}

The polymorphic phase transition of 1,2,4,5-tetrachloroben-

Received 19 October 2005 zene (TCB) has been investigated using neutron powder diffraction and single-crystal $\mathrm{X}$-ray diffraction. The diffraction experiments show a reversible phase change that occurs as a function of temperature with no apparent loss of sample quality on transition between the two phases. Neutron powder diffraction gives detailed information on the molecular structural changes and lattice parameters from $2 \mathrm{~K}$ to room temperature. The structure of the low-temperature form has been elucidated for the first time using single-crystal X-ray diffraction. Comparison of the $\alpha$ and $\beta$ structures show that they are both based on the same sheet motif, with the differences between the two being very subtle, except in terms of crystal symmetry. Detailed analysis of the structures revealed the changes required for inter-conversion. A computational polymorph search showed that these two sheet structures are more thermodynamically stable than alternative herringbone-type structures.

\section{Introduction}

Polymorphism, the ability of a compound to crystallize in more than one crystal structure, is a topic of great interest for both academic research and many industries as it has the potential to significantly affect the physical properties of the compound (Bernstein, 2002). A detailed analysis of the different polymorphic forms adopted by a compound can yield a structural basis for these physical property differences, which often have important ramifications; indeed, there are many documented cases where production of (or transition to) a polymorph other than that desired has led to severe problems in the manufacture or use of particular compounds (Chemburkar et al., 2000). The field of crystal structure prediction aims to allow many of these problems to be anticipated in advance (Price, 2004), particularly by identifying cases where there is a possibility of finding a structure that is more stable than the known forms (Jetti et al., 2003).

Of particular interest are compounds with polymorphic forms that exhibit a phase transition on changing environmental conditions, e.g. temperature, pressure etc.; the relationship between the two forms must be such that they can interconvert without the need to pass through a different physical state. Tetrachlorobenzene (TCB) is one such compound; the low-temperature $\alpha$ form transforms upon warming to the room-temperature $\beta$ form. Although the $\beta$ form has been studied and the structure determined (Herbstein, 1965; Anderson et al., 1991), only the cell dimensions of the $\alpha$ form had been reported prior to this work and the similarity in the cell dimensions of both forms suggested that
Accepted 15 December 2005
(C) 2006 International Union of Crystallography Printed in Great Britain - all rights reserved 
Table 1

Crystal data for the long neutron powder data collections (2, 150, 200 and $295 \mathrm{~K})$ and the single-crystal X-ray data collections.

Note: the non-standard setting has been used for the $\alpha$ phase to allow a direct comparison to the $\beta$ phase; published X-ray data for the $\beta$ phase at $173 \mathrm{~K}$ included for reference (Anderson et al., 1991).

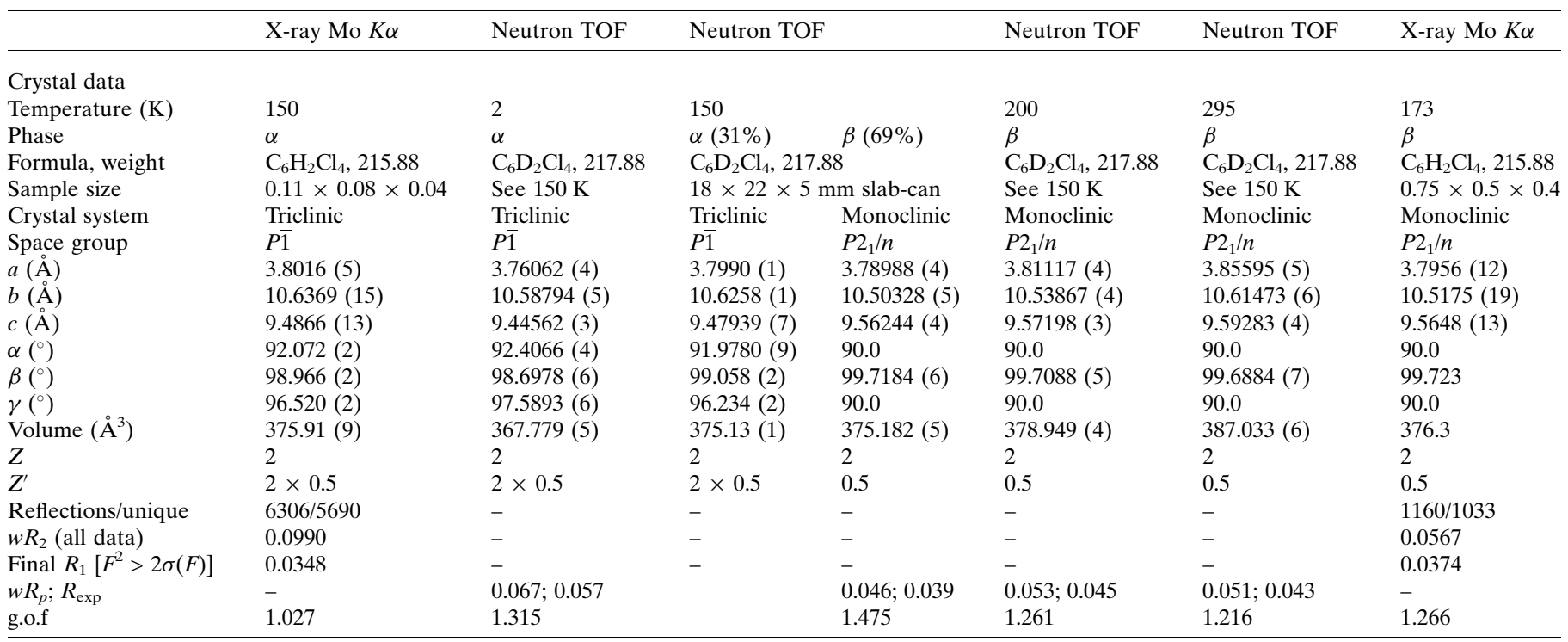

the structures are closely related (Herbstein, 1965; Halac et al., 1977).

It is comparatively rare to be able to observe solid-to-solid phase transitions in a single-crystal sample without an accompanying change to a polycrystalline state. Accordingly, a study of the phase transition in TCB as a function of temperature has been performed using both neutron powder diffraction and single-crystal X-ray diffraction.

\section{Experimental}

1,2,4,5-Tetrachlorobenzene $\left(\mathrm{C}_{6} \mathrm{H}_{2} \mathrm{Cl}_{4}\right)$ was purchased from Aldrich Chemicals and recrystallized from a range of solvents using various methods (see supplementary material ${ }^{\mathbf{1}}$ ). The crystals obtained were predominantly colourless needles and laths. Deuterated tetrachlorobenzene $\left(\mathrm{C}_{6} \mathrm{D}_{2} \mathrm{Cl}_{4}\right)$ was purchased from QMX and used for neutron powder diffraction studies without further purification.

\subsection{Single-crystal $X$-ray diffraction}

All single-crystal X-ray experiments were performed on a Bruker AXS SMART APEX CCD detector diffractometer equipped with a Bruker AXS Kryoflex open-flow cryostat [graphite-monochromated Mo $K \alpha$ radiation $(\lambda=0.71073 \AA)$; $\omega$ scans]. Other details of crystal data, data collection and processing are given in Table 1 and the supplementary material.

Data were collected on a crystal at $200 \mathrm{~K}$, which indexed to be the $\beta$ form. The crystal was then cooled slowly to $150 \mathrm{~K}$ and, somewhat surprisingly, remained intact through the phase

\footnotetext{
${ }^{1}$ Supplementary data for this paper are available from the IUCr electronic archives (Reference: SO5001). Services for accessing these data are described at the back of the journal.
}

transition to the $\alpha$ form. It was evident, through the use of RLATT (Bruker AXS Inc., 2000), that the crystal was twinned (non-merohedral) both before and after the phase transition. GEMINI (Bruker AXS Inc., 2000) was used to index the data and implied that there were two, approximately equal, components. The $150 \mathrm{~K}$ data collected for the $\alpha$ form were integrated twice using each orientation matrix and TWINHKL, within GEMINI, was used to write two data files, one containing only non-overlapping data for one component and one containing all data derived from one domain. The single-crystal structure was solved by direct methods using SHELXS97 (Sheldrick, 1997) on just a single component and all non-H atoms were located using subsequent differenceFourier methods in SHELXL97 (Sheldrick, 1997). H atoms were placed in calculated positions and thereafter allowed to ride on their parent atoms. However, the data completeness was only $80.4 \%$ so the latter data file was used $(98.7 \%)$. The twin components were related by the twin law $\left(\begin{array}{lll}-1 & 0-0.08 / 0\end{array}\right.$ $-1-0.06 / 001$ ) and were in the ratio 30:70.

Several other crystals were cooled slowly (1-2 K steps) from 200 to $150 \mathrm{~K}$ and a single frame collected at the diffractometer zero position for each temperature in order to observe the range of temperatures over which the phase transition occurred. Once the phase transition appeared complete, the crystal sample was slowly warmed back to $200 \mathrm{~K}$. Unit-cell dimensions were determined at $200 \mathrm{~K}$, both before and after the phase transition, as well as at $150 \mathrm{~K}$, to identify which phases were present.

\subsection{Neutron powder diffraction}

Neutron powder diffraction experiments were carried out on the HRPD instrument at the ISIS Facility of the CCLRC Rutherford Appleton Laboratory, Oxfordshire, England. 
A perdeuterated sample ( $c a 1 \mathrm{~g}$ ) of the $\beta$ phase of TCB was placed in a $5 \mathrm{~mm}$ flat vanadium can, cooled through the phase transition to give the $\alpha$ form, and a long data collection ( $c a$ $4 \mathrm{~h}$ ) was carried out at $2 \mathrm{~K}$ to provide data suitable for structure refinement. The sample was then warmed in $2 \mathrm{~K}$ steps with a short $(10 \mathrm{~min})$ data collection at each step; transformation from $\alpha$ to $\beta$ was observed over the temperature range $154-182 \mathrm{~K}$. At $295 \mathrm{~K}$, another long data collection ( ca $5 \frac{1}{2} \mathrm{~h}$ ) was carried out before the sample was cooled back down to the region of the phase transition and further long data collections carried out at $200 \mathrm{~K}$ and $150 \mathrm{~K}$ ( $c a 5 \frac{1}{2}$ and $8 \mathrm{~h}$, respectively). The sample exhibited significant hysteresis and the transformation back to the $\alpha$ form was incomplete by $150 \mathrm{~K}$, with the result that the $150 \mathrm{~K}$ data represents a mixed phase.

Initial structures were taken from the relevant single-crystal $\mathrm{X}$-ray models and structure refinement carried out using a restrained Rietveld method (Rietveld, 1969) as implemented in TOPAS (Coelho, 2003). In the full structural refinements (i.e. the long data collections) atomic coordinates were refined (subject to a series of geometric restraints) along with the lattice constants, background, peak shape and preferred orientation parameters. In the case of the $150 \mathrm{~K}$ data, the $\alpha: \beta$ phase ratio was also refined, but the coordinates of the $\alpha$ phase were constrained as a rigid body that was free to rotate. All atoms were refined isotropically, with all atoms of the same type (within a single phase and refinement) constrained to have equal $B_{\text {iso }}$ values.

For the refinements against the data from the short data collections (warming cycle), only the lattice parameters, background, peak-shape parameters and, where appropriate, $\alpha: \beta$ phase ratio were refined. The atomic coordinates were taken from either the $2 \mathrm{~K}$ ( $\alpha$ phase), $295 \mathrm{~K}$ ( $\beta$ phase) or the $150 \mathrm{~K}$ (mixed phase) refinement.

\subsection{Structure prediction calculations}

A gas-phase model for the tetrachlorobenzene molecule was obtained by optimization of the MP2/6-31G** energy using the program GAUSSIAN98 (Frisch et al., 1998). The corresponding wavefunction was also calculated for the X-ray determined molecular structures, with the $\mathrm{C}-\mathrm{H}$ bond length
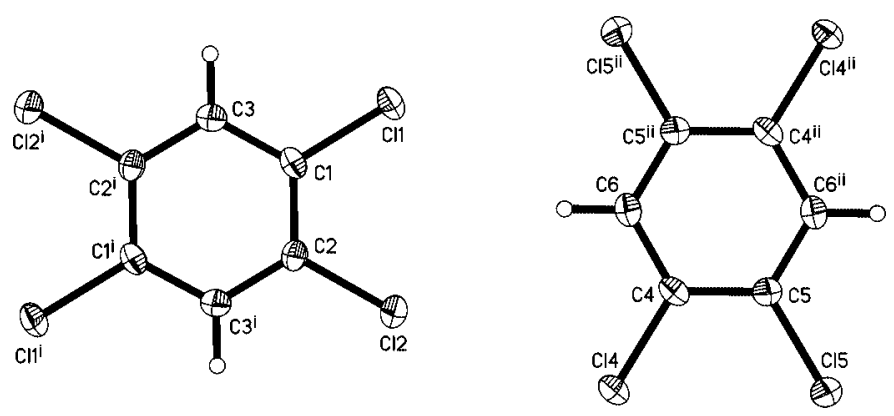

Figure 1

The numbering scheme used for the $\alpha$ form of 1,2,4,5-tetrachlorobenzene. Displacement ellipsoids drawn at the $50 \%$ probability level. Symmetry codes: (i) $-x, 1-y, 1-z$; (ii) $1-x,-y,-z$. elongated to the standard neutron value of $1.081 \AA$ (Allen et al., 1987). A distributed multipole analysis (DMA; Stone \& Alderton, 1985) of the ab initio charge density of the molecule was performed to provide an accurate description of the electrostatic contribution to the lattice energy in the rigidmolecule crystal structure modeling. This atomic multipolar electrostatic model automatically represents the electrostatic effects of lone pair and $\pi$ electron density (Price, 1996).

However, it has been observed that the crystal packing of chlorinated organic molecules, which results in an anisotropic van der Waals radius for $\mathrm{Cl}$, does not only arise from the anisotropy in the electrostatic interaction, but also from the effect of lone-pair density giving an anisotropic repulsive wall (Price et al., 1994). Thus, a non-empirical model potential developed specifically for the chlorobenzene crystal structures (Day \& Price, 2003), which is able to reproduce a wide range of the properties of the known chlorobenzenes crystal structures, was used.

The hypothetical crystal structures for tetrachlorobenzene were generated by MOLPAK (Holden et al., 1993), which performs a systematic grid search on orientations of the rigid central molecule in 29 common coordination geometries of organic molecules, belonging to the space groups $P 1, P \overline{1}, P 2_{1}$, $P 2_{1} / c, C 2, C c, C 2 / c, P 2_{1} 2_{1} 2, P 2_{1} 2_{1} 2_{1}, P c a 2_{1}, P n a 2_{1}, P b c n$ and $\mathrm{Pbca}$, with one molecule in the asymmetric unit. Approximately 50 of the densest packings in each coordination type are then used as starting points for lattice energy minimization by DMAREL (Willock et al., 1995) using the non-empirical atom-atom based model potential.

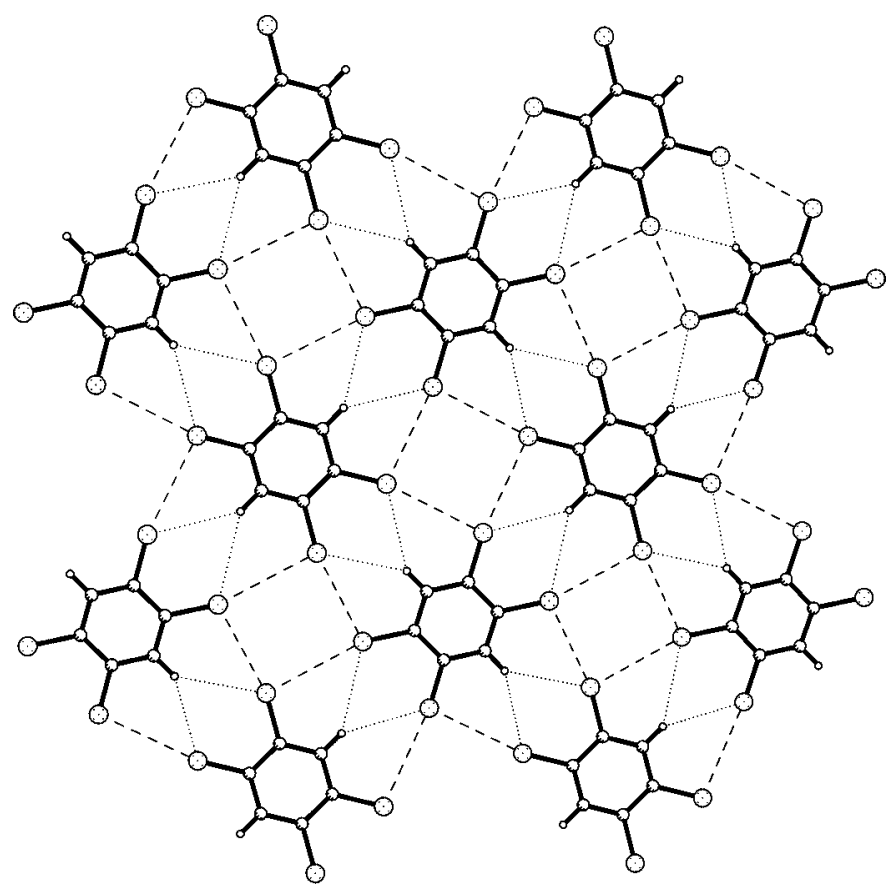

Figure 2

The two-dimensional sheet formed by the $\alpha$ form of 1,2,4,5-tetrachlorobenzene viewed perpendicular to the $\left[\begin{array}{lll}2 & 0 & 2\end{array}\right]$ plane showing the $\mathrm{Cl} \cdots \mathrm{Cl}$ interactions (dashed) and bifurcated hydrogen bonds (dotted). C shaded; $\mathrm{Cl}$ - dotted. 
Table 2

The effect of the phase transition on the lattice parameters .

The absolute change in value from the $\beta$ to the $\alpha$ phase is given as well as this change as a percentage of the $\alpha$ lattice parameter. The absolute shifts in the lattice parameters are small.

\begin{tabular}{lllll}
\hline & & & \multicolumn{2}{c}{ Lattice parameter shift at phase transition } \\
\cline { 5 - 5 } & $\alpha(150 \mathrm{~K})$ & $\beta(150 \mathrm{~K})$ & Absolute & Relative to $\alpha$ phase \\
\hline$a(\AA)$ & $3.7990(1)$ & $3.78988(4)$ & -0.0091 & $-0.24 \%$ \\
$b(\AA)$ & $10.6258(1)$ & $10.50328(5)$ & -0.12 & $-1.2 \%$ \\
$c(\AA)$ & $9.47939(7)$ & $9.56244(3)$ & 0.083 & $0.88 \%$ \\
$\alpha\left({ }^{\circ}\right)$ & $91.9780(9)$ & 90.0 & -2.0 & $-2.2 \%$ \\
$\beta\left({ }^{\circ}\right)$ & $99.058(2)$ & $99.7184(6)$ & 0.66 & $0.67 \%$ \\
$\gamma\left({ }^{\circ}\right)$ & $96.234(2)$ & 90.0 & -6.2 & $-6.5 \%$ \\
Volume $\left(\AA^{3}\right)$ & $375.13(1)$ & $375.182(5)$ & 0.052 & $0.014 \%$ \\
\hline
\end{tabular}

The second derivative properties of each lattice energy minimum were examined (Day et al., 2001) and those that were mechanically unstable were eliminated. The distinct lowenergy minima within $20 \mathrm{~kJ} \mathrm{~mol}^{-1}$ of the global minimum were established by considering the reduced cell parameters (Křivý, 1976) using PLATON (Spek, 2003) and clustering the identical structures. As the MOLPAK search only produces structures with one entire molecule in the asymmetric unit of the space groups considered, the crystal structures lying within $5 \mathrm{~kJ} \mathrm{~mol}^{-1}$ of the global minimum were run through the ADDSYM function of PLATON in order to correct the symmetry for structures where the true $Z^{\prime}$ was less than 1 and obtain a list of 17 unique structures.

\section{Results and discussion}

\subsection{Determination of the low-temperature $a$ poly- morph}

Crystals of TCB, prepared by a variety of crystallization methods using a number of different solvents (see Table 1 of the supplementary material), tended to be of two types: colourless laths and needles, of which, the laths were better suited to single-crystal X-ray diffraction. A large number of these crystals (both laths and needles) were placed on the diffractometer at $200 \mathrm{~K}$ and all, without exception, indexed as the $\beta$ form. One of the crystals (a 30:70 non-merohedral twinned crystal) was cooled slowly using the Kryoflex in $\sim 2.5 \mathrm{~K}$ steps from 200 to $190 \mathrm{~K}, \sim 1 \mathrm{~K}$ steps to $182 \mathrm{~K}, 2 \mathrm{~K}$ steps to $170 \mathrm{~K}$ and $5 \mathrm{~K}$ steps to $150 \mathrm{~K}$ with

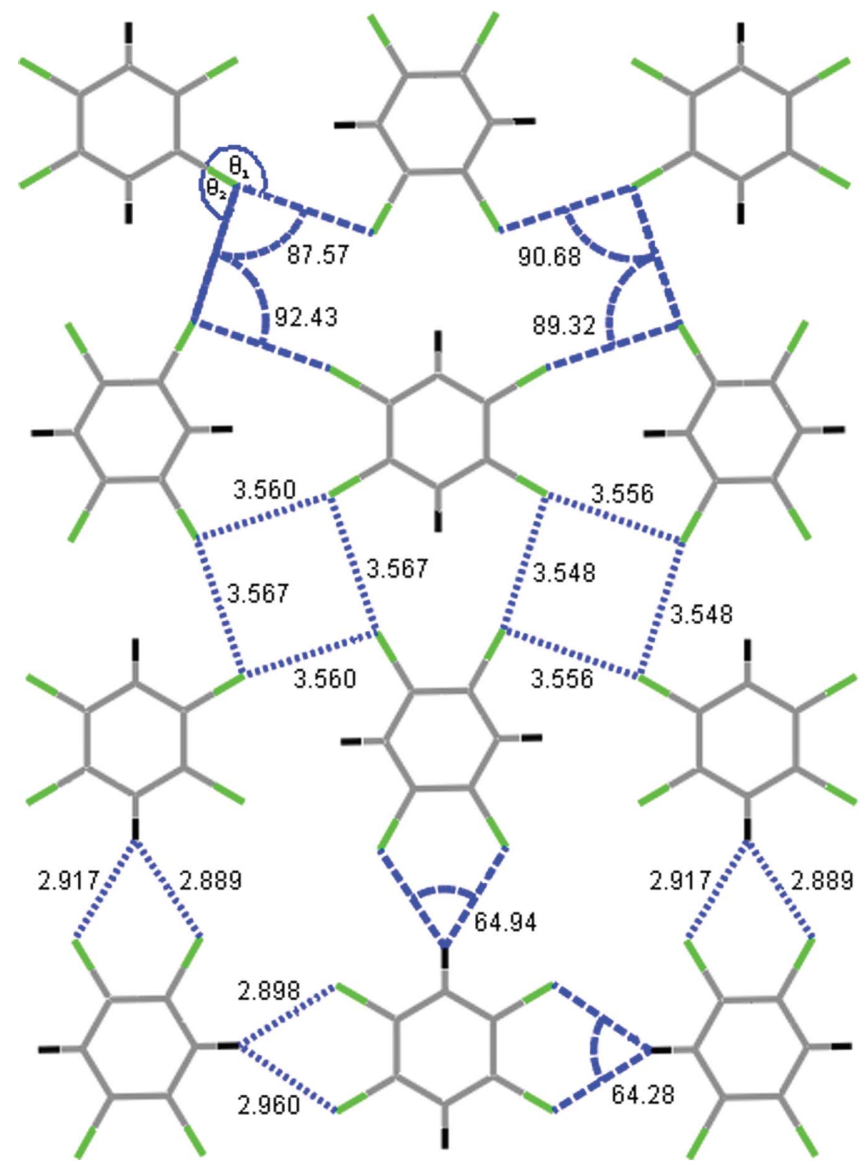

(a)

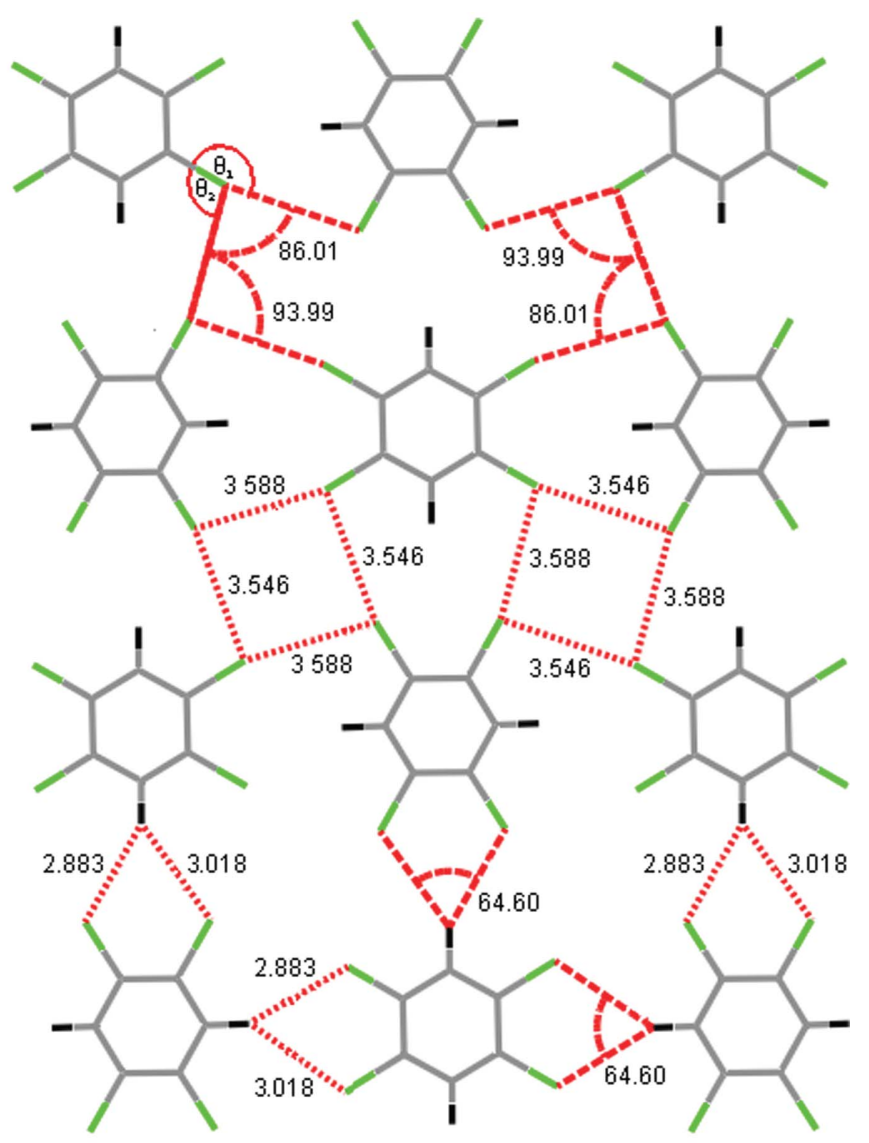

(b)

Figure 3

The bond lengths $(\AA)$ and angles $\left({ }^{\circ}\right)$ of the $\mathrm{Cl} \cdots \mathrm{Cl}$ interactions and bifurcated hydrogen bonds found in $(a)$ the $\alpha$ form and $(b)$ the $\beta$ form. 
Table 3

Geometry of the tetrameric $\mathrm{Cl} \cdots \mathrm{Cl}$ interaction, and the bifurcated hydrogen bonds.

Values taken from the powder data model at $150 \mathrm{~K}$ (single-crystal data values are similar).

\begin{tabular}{lllllll}
\hline & Interaction & $\mathrm{Cl} \cdots \mathrm{Cl}(\AA)$ & $\mathrm{C}-\mathrm{Cl} \cdots \mathrm{Cl} \theta_{1}\left({ }^{\circ}\right)$ & $\mathrm{Cl} \cdots \mathrm{Cl}-\mathrm{C} \theta_{2}\left(^{\circ}\right)$ & Internal angle $\left(^{\circ}\right)$ & \\
\hline \multirow{2}{*}{$\alpha$ phase } & $\mathrm{Cl} 5 \cdots \mathrm{Cl} 8$ & 3.578 & 165.41 & 100.50 & $\mathrm{Cl} 5 \cdots \mathrm{Cl} 8 \cdots \mathrm{Cl} 5$ & 90.69 \\
& $\mathrm{Cl} 8 \cdots \mathrm{Cl} 5$ & 3.531 & 156.39 & 101.30 & $\mathrm{Cl} 8 \cdots \mathrm{Cl} 5 \cdots \mathrm{Cl} 8$ & 89.31 \\
& $\mathrm{Cl} 6 \cdots \mathrm{Cl} 9$ & 3.545 & 161.45 & 100.19 & $\mathrm{Cl} 6 \cdots \mathrm{Cl} 9 \cdots \mathrm{Cl} 6$ & 87.57 \\
& $\mathrm{Cl} 9 \cdots \mathrm{Cl} 6$ & 3.572 & 161.84 & 100.47 & $\mathrm{Cl} 9 \mathrm{Cl} 6 \cdots \mathrm{Cl} 9$ & 92.43 \\
$\beta$ phase & $\mathrm{Cl} \cdots \mathrm{Cl} 2$ & 3.538 & 164.83 & 101.63 & $\mathrm{Cl} 1 \cdots \mathrm{Cl} 2 \cdots \mathrm{Cl} 1$ & 93.89 \\
& $\mathrm{Cl} 2 \cdots \mathrm{Cl} 1$ & 3.578 & 151.95 & 102.65 & $\mathrm{Cl} 2 \cdots \mathrm{Cl} 1 \cdots \mathrm{Cl} 2$ & 86.11 \\
\hline
\end{tabular}

\begin{tabular}{|c|c|c|c|c|c|}
\hline & $\mathrm{H} \cdots \mathrm{Cl}_{a}(\AA)$ & $\mathrm{H} \cdots \mathrm{Cl}_{b}(\AA)$ & $\mathrm{C}-\mathrm{H} \cdots \mathrm{Cl}_{a}\left({ }^{\circ}\right)$ & $\mathrm{C}-\mathrm{H} \cdots \mathrm{Cl}_{b}\left({ }^{\circ}\right)$ & $\mathrm{Cl}_{a} \cdots \mathrm{H} \cdots \mathrm{Cl}_{b}\left({ }^{\circ}\right)$ \\
\hline \multicolumn{6}{|c|}{ Bifurcated bond } \\
\hline \multirow[t]{2}{*}{$\alpha$ phase } & 2.940 & 2.909 & 141.35 & 144.85 & 64.41 \\
\hline & 2.927 & 2.896 & 146.92 & 144.93 & 64.73 \\
\hline$\beta$ phase & 2.883 & 3.011 & 147.34 & 143.84 & 64.99 \\
\hline
\end{tabular}

image frames collected at each step in the diffractometer zero position. Slight changes, attributable to thermal contraction, were observed between successive images, except between 175 and $172 \mathrm{~K}$ where there was a major change in the diffraction pattern due to the transition from the monoclinic $\beta$ form to the triclinic $\alpha$ form. The structure of the $\alpha$ form was solved on a full data set collected at $150 \mathrm{~K}$.

The $\alpha$ form of TCB crystallizes in the space group $P \overline{1}$ with two half molecules sitting on inversion centres in the asymmetric unit (Fig. 1; see Table 2 of the supplementary material for selected bond lengths and angles). Each molecule is involved in four $\mathrm{Cl} \cdots \mathrm{Cl}$ interactions and two bifurcated hydrogen bonds (see $\$ 3.3$ for full structural analysis and comparison to the $\beta$ phase) to build up two-dimensional sheets which stack as the [2 02 2] Miller planes, each separated by $3.28 \AA$ (Fig. 2). There are $\pi-\pi$ stacking interactions between TCB molecules in adjacent planes (centroid-centroid distance $3.799 \AA \equiv$ length of $a$ axis; offset $1.58 \AA$ ). This structure is very

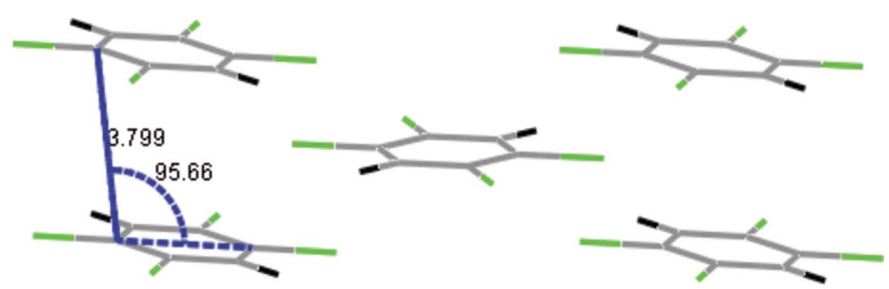

(a)

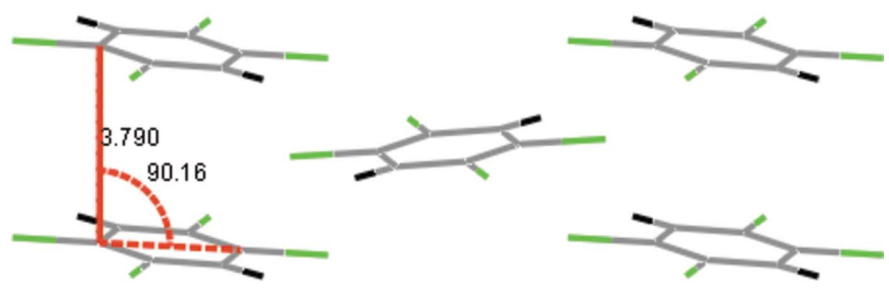

(b)

Figure 4

The distance $(\AA)$ and angle $\left({ }^{\circ}\right)$ between stacked molecules in $(a)$ the $\alpha$ form and $(b)$ the $\beta$ form, as viewed down the $c$ axis. similar to that found for the roomtemperature $\beta$ form, since the two polymorphs exhibit isomorphic sheet structures of TCB molecules which stack parallel to the [lll $\left.\begin{array}{lll}2 & 2\end{array}\right]$ Miller plane.

\subsection{Structural refinement from neutron powder data}

Good agreement was found between the structures obtained from single-crystal X-ray data and those refined from the neutron powder data. For further details of the structures at 2, 150, 200 and $295 \mathrm{~K}$, including CIFs, see the supplementary material. The incomplete phase change seen during the cooling cycle resulted in the coexistence of both phases $(31 \% \alpha, 69 \% \beta)$ in the $150 \mathrm{~K}$ 'long' data set, presenting the opportunity for direct structural comparison under identical conditions. The change in lattice parameters at the phase transition cancel out in such a way that the change in volume is almost negligible; $V(\alpha)=$ 375.13 (1), $V(\beta)=375.182$ (5) $\AA^{3}$ (see Table 2). The absolute changes in the values of the lattice parameters at the phase transition are very small, with the largest shift in unit-cell axis length being only $0.12 \AA(\sim 1 \%)$. The only shift of any appreciable size is that of the $\gamma$ angle, which changes by $6.2^{\circ}$. The phase transition is discussed further in $§ 3.5$.

\subsection{Structural comparison of $\alpha$ and $\beta$ phases}

The two phases of tetrachlorobenzene are remarkably similar in structure, although quite distinct crystallographically. The $\beta$ form crystallizes in the monoclinic space group $P 2_{1} / n$ with $Z=2$ and $Z^{\prime}=\frac{1}{2}$, whereas, the $\alpha$ form crystallizes in $P \overline{1}$, again with $Z=2$, but with two independent half molecules in the asymmetric unit. Both forms are characterized by sheets of tetrachlorobenzene molecules that lie in the $\left[\begin{array}{lll}2 & 0 & 2\end{array}\right]$ plane with an inter-plane distance of $3.28 \AA$, and are arranged such that the ring centroids are separated by the length of the $a$ axis. Comparison of an individual sheet from each form reveals very little difference between the two (Fig. 3 , Table 3) with the molecules within the sheets taking part in an extended series of $\mathrm{Cl} \cdots \mathrm{Cl}$ interactions. Since there are two independent molecules in the asymmetric unit of the $\alpha$ form, there are two crystallographically different sets of $\mathrm{Cl} \cdots \mathrm{Cl}$ interactions compared with just one for the $\beta$ form. The $\mathrm{C}-$ $\mathrm{Cl} \cdots \mathrm{Cl}-\mathrm{C}$ interaction is characterized by two unequal $\mathrm{C}-$ $\mathrm{Cl} \cdots \mathrm{Cl}$ angles, $\theta_{1}$ and $\theta_{2}$, where $\theta_{1}>\theta_{2}$, and $\theta_{1} \simeq 180^{\circ}$ and $\theta_{2} \simeq$ $90^{\circ}$. This clearly illustrates the anisotropic charge distribution of the carbon-bound $\mathrm{Cl}$ atom (Broder et al., 2000; Price et al., 1994) (see Table 3 for actual values of $\theta_{1}$ and $\theta_{2}$ ). In both phases the $\mathrm{Cl} \cdots \mathrm{Cl}$ interactions form a tetrameric motif centred about an inversion centre, thus the four $\mathrm{Cl}$ atoms of the tetrameric motif are, by definition, planar, with the motifs being more symmetric in the $\alpha$ phase than in the $\beta$ phase. Both 
Table 4

A summary of the unique predicted crystal structures lying within $5 \mathrm{~kJ} \mathrm{~mol}^{-1}$ of the global minimum after correction for missing symmetry.

Similar structures are labelled as having the same packing type, where $\mathrm{S}=$ sheet and $\mathrm{H}=$ herringbone.

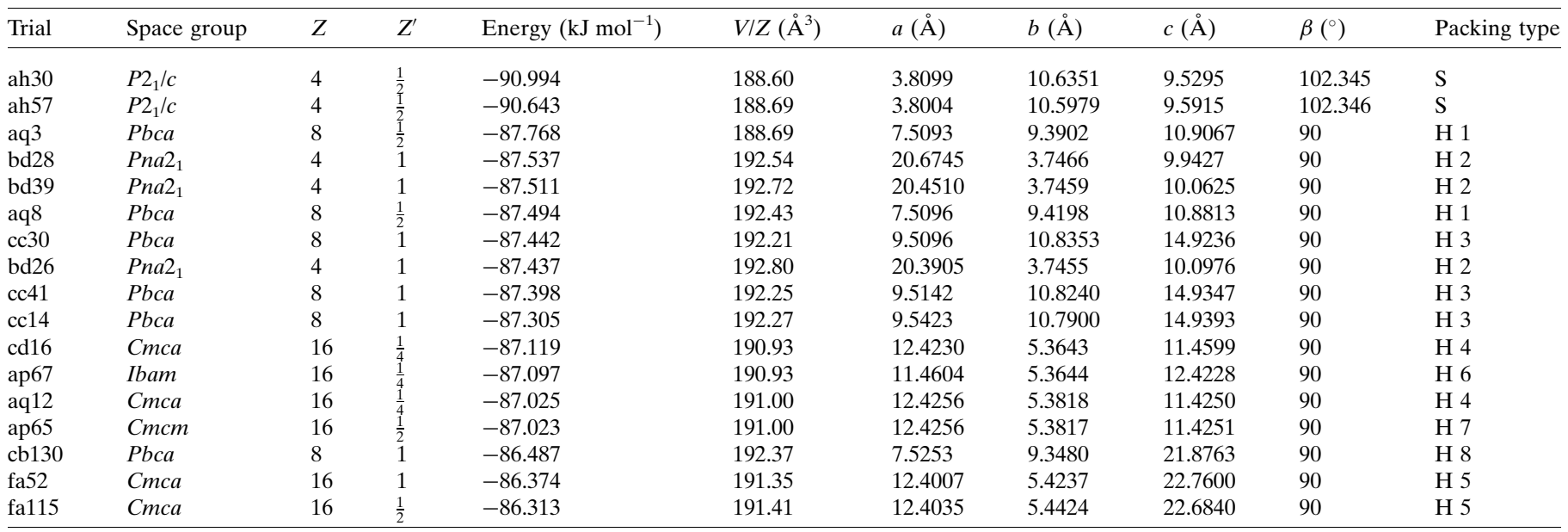

phases also exhibit a bifurcated hydrogen bond (Jeffrey, 1997; Yang \& Gellman, 1998) between the $\mathrm{H}$ atom and two $\mathrm{Cl}$ atoms on an adjacent molecule. The bifurcated hydrogen bonds in the $\alpha$ phase are more symmetric than the same bonds in the $\beta$ phase; see Table 3 for full details. The combination of the

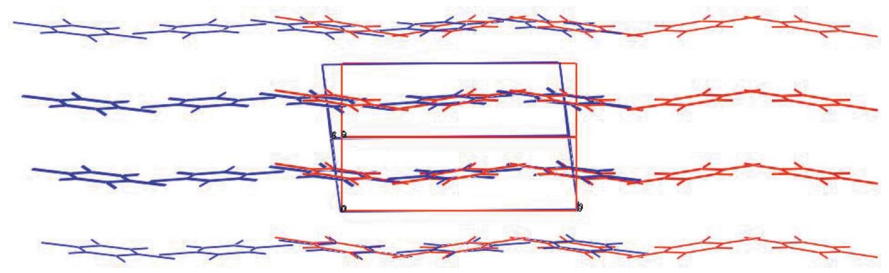

Figure 5

Overlay of the structures of the $\alpha$ phase (blue) and the $\beta$ phase (red) as viewed in projection, perpendicular to the $b$ axes.

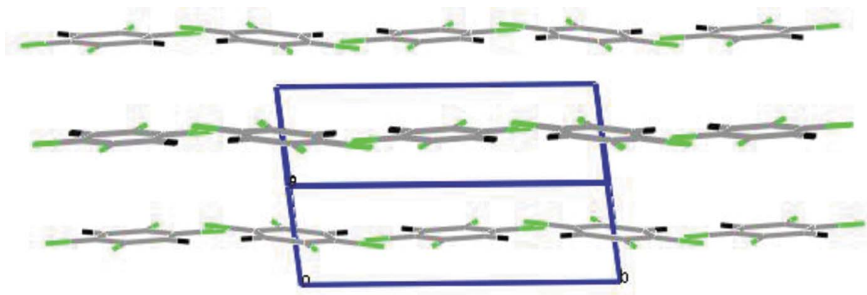

(a)

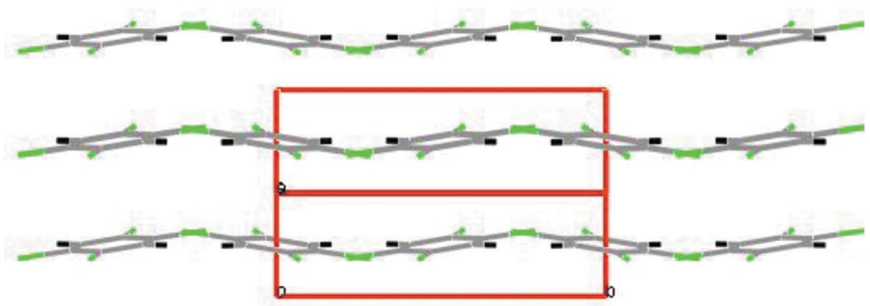

(b)

Figure 6

The sheets in the $(a) \alpha$ and $(b) \beta$ phases viewed in projection, perpendicular to the $b$ axes.
$\mathrm{Cl} \cdots \mathrm{Cl}$ interactions and bifurcated hydrogen bonds results in a very well defined sheet which is retained through the phase transition, with the differences between the polymorphs arising from subtle differences in the way that these sheets stack.

The orientation of the molecules with respect to the [l 202 2] plane is subtly different between the $\alpha$ form and the $\beta$ form. This is shown in Figs. 4, 5 and 6. In both cases the ring centroids lie on the [lll $\left.\begin{array}{ll}2 & 2\end{array}\right]$ plane. In the $\alpha$ phase the angle between the mean planes of two adjacent and symmetryindependent tetrachlorobenzene molecules is $6.67^{\circ}$ with the angle between the mean planes of each of these molecules and the [l 202 ] plane being 7.71 and $9.73^{\circ}$. In the $\beta$ phase the angle between the mean planes of the same two adjacent (now symmetry related) tetrachlorobenzene molecules is $13.36^{\circ}$ and the angle between each of these planes and the [2 02 2] plane is $10.03^{\circ}$. Although the structures of the individual sheets in both

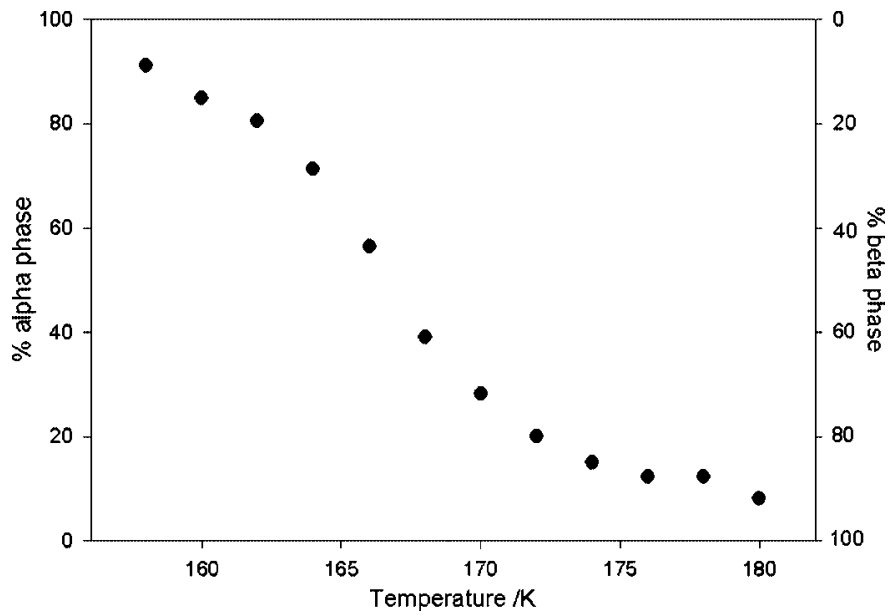

Figure 7

Relative proportion of $\alpha$ to $\beta$ tetrachlorobenzene as a function of temperature for the warming-cycle data sets. 
polymorphs are very similar (Fig. 3), their stacking arrangements are significantly different, as can be seen in Figs. 5 and 6.

When the sheets are viewed side-by-side perpendicular to the $b$ axes, their undulating nature is clearly apparent.
Considering these undulations as waves, the amplitude of the wave is greater in the $\beta$ than in the $\alpha$ form, in which adjacent waves are also slightly offset. The reduction in the amplitude is associated with an increase in periodicity of the wave, i.e. the $b$ axis increases from 10.50328 (5) $\AA$ in the $\beta$ form to 10.6258 (1) $\AA$ in the $\alpha$ form at $150 \mathrm{~K}$. The reduction of the amplitude facilitates movement of adjacent sheets relative to one another, giving rise to an offset in the $\alpha$ form and a change in the $\alpha$ and $\gamma$ angles associated with the loss of symmetry from $P 2_{1} / n$ to $P \overline{1}$.

\subsection{Phase transition tempera- ture range}

The $\alpha: \beta$ ratios calculated from the short neutron powder data collections are plotted in Fig. 7. It can be seen that the phase transition occurs smoothly and over a significant time and temperature range. Whilst fully reversible, there is a large hysteresis loop associated with the phase transition; on warming, the phase change occurs between 154 and $182 \mathrm{~K}$, yet on cooling the phase transition is incomplete at $150 \mathrm{~K}$. The phase transition was also observed using single-crystal $\mathrm{X}$ ray diffraction, with the precise temperature range of the transition being sample dependent. On cooling four multi-domain twinned-crystal samples, the $\alpha$ form started to appear between 172 and $170 \mathrm{~K}$ with the phase transition complete by $160 \mathrm{~K}$; on warming back up to $200 \mathrm{~K}, \beta$ starts to appear in the temperature range $165-175 \mathrm{~K}$ and the transition is complete at $184 \mathrm{~K}$. Interestingly, this transition was only observed in twinned crystals; a non-twinned single-crystal sample of the $\beta$ phase showed no transition to the $\alpha$ phase in accordance with other observations (Mnyukh, 2001). In a twodomain 30:70 twinned crystal the number and size of the domains remained

\section{Figure 8}

Lattice parameters of $\alpha$ and $\beta$ phase tetrachlorobenzene (blue and red, respectively) from 2 to $295 \mathrm{~K}$. Circles represent data from the short data collections (warming cycle) while diamonds represent long data collections (cooling cycle). 


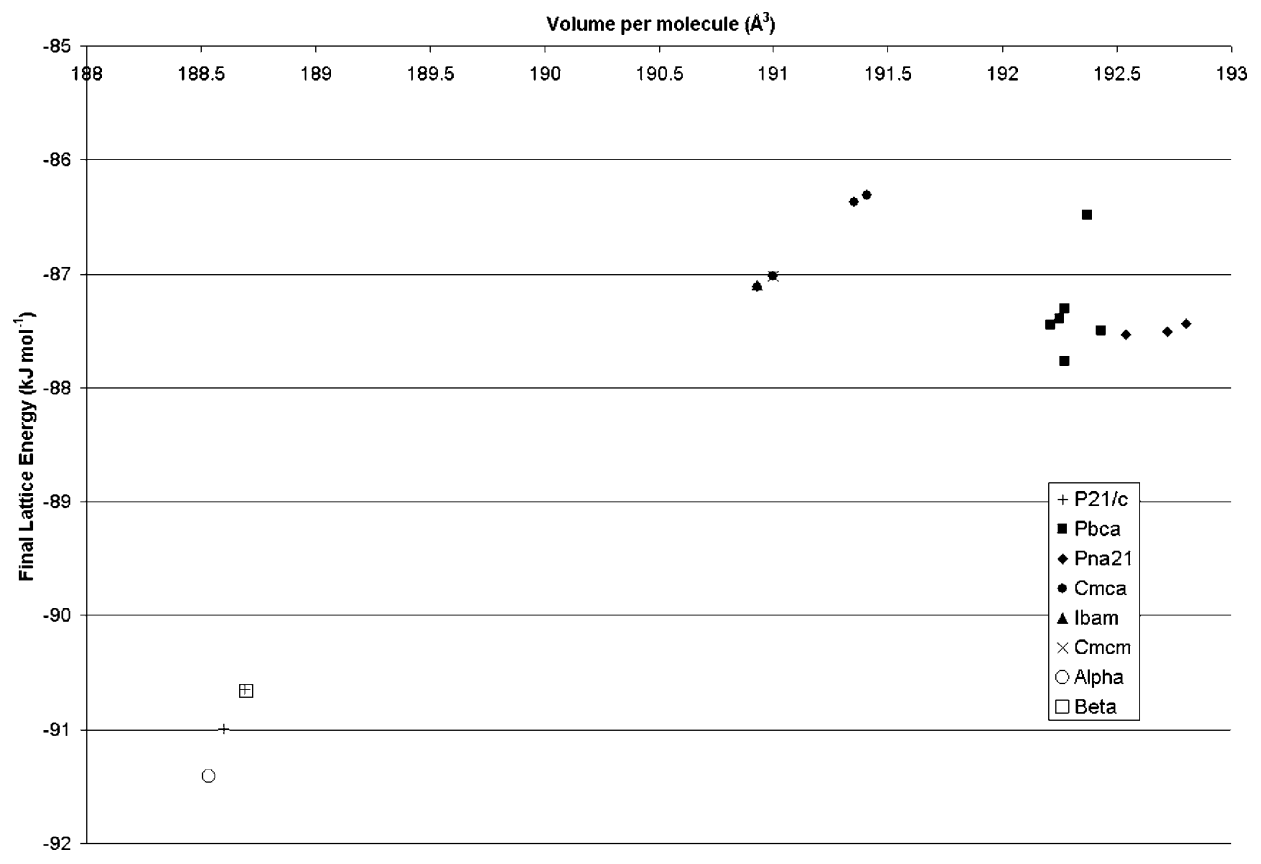

Figure 9

Plot of lattice energy against cell volume per molecule for the unique structures within $5 \mathrm{~kJ} \mathrm{~mol}^{-1}$ of the global minimum denoted by the space group assigned after being run through the ADDSYM function of PLATON. The structures corresponding to the $\alpha$ and $\beta$ forms using the same computational model are also shown.

unchanged by the phase transition and the phase change was sharp (175-172 K). A more gradual phase transition was observed in multi-domain twinned crystals. This implies that the domain boundaries are necessary for phase transition.

\subsection{Thermal expansion and the phase transition}

Fig. 8 shows the variation in lattice parameters with temperature observed for the neutron powder data. The overlap between the $\alpha$ and $\beta$ phase curves (blue and red, respectively) indicates where both phases are present simultaneously in the sample. There is good agreement between the lattice parameters obtained from the short data collections and the long data collections (data points symbolized by circles and diamonds, respectively). The change in volume at the phase transition is negligible; on visual inspection, the rate of thermal expansion in volume appears almost continuous across the phase transition. ${ }^{2}$ The length of the $a$ axis, which corresponds to the distance between adjacent aromatic ring centroids, also hardly varies at the phase transition, shifting by only $0.0091 \AA$; however, this shift is significant being greater than 3 times the e.s.d. (0.0001 $\AA$ ). The $b$ and $c$ axes exhibit a slightly larger shift at the phase transition. The $\gamma$ angle undergoes the largest shift, $6.2^{\circ}$, at the phase transition; the next largest change is in the $\alpha$ angle. These two changes combined affect the offsetting of the undulating sheets.

${ }^{2}$ Full analysis and fit of these graphs to be published in a separate article.

\subsection{Structural prediction calculations}

The search for crystal structures corresponding to the minima in the lattice energy produced approximately 350 mechanically stable structures within $10 \mathrm{~kJ} \mathrm{~mol}^{-1}$ of the global minimum. The resulting unique structures, within $5 \mathrm{~kJ} \mathrm{~mol}^{-1}$, are shown in Table 4 and Fig. 9. The two lowest energy structures (ah30 and ah57) are essentially identical, indicating a complicated energy surface, and have unit cells corresponding to that of the $\beta$ form of TCB (transforming ah30 into $P 2_{1} / n$ gives a unit cell of $a=3.8099, b=$ 10.6351, $c=9.4764 \AA \quad \beta=$ $100.78^{\circ}$ ). After transformation, both these predicted structures are virtually superimposable with the structure obtained from the single-crystal literature. The $\alpha$ form $\left(Z^{\prime}=2 \times \frac{1}{2}\right)$ is not predicted in this search because of limitations in the search method, which mean that structures with $Z^{\prime}>1$ could not be screened. However, a lattice-energy minimization starting from the $\alpha$ structure gives a minimum that is only slightly $\left(0.75 \mathrm{~kJ} \mathrm{~mol}^{-1}\right)$ more stable than the $\beta$ form. There is a significant energy gap of $3 \mathrm{~kJ} \mathrm{~mol}^{-1}$ between these two structures and the next lowest-energy structure, which indicates that other polymorphs are unlikely to be found. As the known structures are the densest, it is unlikely that highpressure polymorphs exist, although this does not exclude the possible existence of high-temperature phases, as thermally induced motion could result in an entropic term great enough to overcome this $3 \mathrm{~kJ} \mathrm{~mol}^{-1}$ gap.

\section{Conclusions}

Below room temperature there are no additional new lowtemperature phases that are accessible simply by changing the temperature. The phase transition is observed over a temperature range and the precise values of this temperature range appear to be sample and sample-history dependent. However, all the samples exhibited a hysteresis loop with the phase transition temperature consistently lower on cooling from $\beta$ to $\alpha$ than on warming from $\alpha$ to $\beta$. The short-scan neutron powder diffraction data revealed that the phase transition from $\alpha$ to $\beta$ is gradual for a powder sample and that a temperature range exists where both phases are simultaneously present. Further single-crystal work proved that a gradual phase transition was observed in multi-domain 
twinned crystals, whereas, in two-domain twinned crystals the phase transition was much sharper and no phase transition was observed in single-domain (non-twinned) crystals. The three largest shifts in lattice parameter on going from the $\beta$ to the $\alpha$ form of tetrachlorobenzene can by understood by considering the two phases as stacks of undulating sheets with the changes in lattice parameters occurring as a result of the effect of offsetting these sheets. It is usually expected that there will be a loss of symmetry on cooling and indeed this system goes from $P 2_{1} / n$ to $P \overline{1}$ on cooling and, in addition, the number of molecular units in the asymmetric unit doubles. However, internally the $\alpha$ polymorph could be considered as being the more perfect system with less of a tilt between adjacent molecules and more symmetric bifurcated hydrogen bonds and $\mathrm{Cl}$.. Cl tetramers. The computational search confirms that these two polymorphs are very close in energy, as well as structure, and more stable than other, alternative packings.

The authors would like to acknowledge the Research Councils UK Basic Technology Programme for supporting 'Control and Prediction of the Organic Solid State' (http:// www.cposs.org.uk). Thanks go to Professor S. L. Price (University College London) for helpful discussions, particularly with regards to the structural prediction work.

\section{References}

Allen, F. H., Kennard, O. \& Watson, D. G. (1987). J. Chem. Soc. Perkin Trans. 2, pp. S1-S18.

Anderson, D. G., Blake, A. J., Blom, R., Cradock, S. \& Rankin, D. W. H. (1991). Acta Chem. Scand. 45, 158-164.

Bernstein, J. (2002). Polymorphism in Molecular Crystals. Oxford: Clarendon Press.

Broder, C. K., Howard, J. A. K., Keen, D. A., Wilson, C. C., Allen, F. H., Jetti, R. K. R., Nangia, A. \& Desiraju, G. R. (2000). Acta Cryst. B56, 1080-1084.
Bruker AXS Inc. (2000). Gemini, Version 1.02; RLATT, Version 3.00. Bruker AXS Inc., Madison, Wisconsin, USA.

Chemburkar, S. R., Bauer, J., Deming, K., Spiwek, H., Patel, K., Morris, J., Henry, R., Spanton, S., Dziki, W., Porter, W., Quick, J., Bauer, P., Donaubauer, J., Narayanan, B. A., Soldani, M., Riley, D. \& McFarland, K. (2000). Org. Process Res. Dev. 4, 413-417.

Coelho, A. A. (2003). J. Appl. Cryst. 36, 86-95.

Day, G. M. \& Price, S. L. (2003). J. Am. Chem. Soc. 125, 1643416443.

Day, G. M., Price, S. L. \& Leslie, M. (2001). Cryst. Growth Des. 1, 1326.

Frisch, M. J. et al. (1998). GAUSSIAN98, Revision A.9. Gaussian, Inc., Pittsburgh, PA, USA.

Herbstein, F. H. (1965). Acta Cryst. 18, 997-1000.

Halac, E. B., Burgos, E. M., Bonadeo, H. E. \& D'Alessio, A. (1977). Acta Cryst. A33, 86-89.

Holden, J. R., Du, Z. Y. \& Ammon, H. L. (1993). J. Comput. Chem. 14, 422-437.

Jeffrey, G. A. (1997). An Introduction to Hydrogen Bonding. New York: Oxford University Press.

Jetti, R. K. R., Boese, R., Sarma, J. A. R. P., Reddy, L. S., Vishweshwar, P. \& Desiraju, D. R. (2003). Angew. Chem. Int. Ed. 42, 1963-1967.

Křivý, I. (1976). Acta Cryst. A32, 297-298.

Mnyukh, Y. (2001). Fundamentals of Solid-State Phase Transitions, Ferromagnetism and Ferroelectricity. Bloomington: 1st Books Library.

Price, S. L. (2004). Adv. Drug Delivery Rev. 56, 301-319.

Price, S. L. (1996). J. Chem Soc. Faraday Trans. 92, $2997-$ 3008.

Price, S. L., Stone, A. J., Lucas, J., Rowland, R. S. \& Thornley, A. E. (1994). J. Am. Chem. Soc. 116, 4910-4918.

Rietveld, H. M. (1969). J. Appl. Cryst. 2, 65-71.

Sheldrick, G. M. (1997). SHELXS97 and SHELXL97. University of Göttingen, Germany.

Stone, A. J. \& Alderton, M. (1985). Mol. Phys. 56, 1047-1064.

Spek, A. L. (2003). J. Appl. Cryst. 36, 7-13.

Willock, D. J., Price, S. L., Leslie, M. \& Catlow, C. R. A. (1995). J. Comput. Chem. 16, 628-647.

Yang, Y. \& Gellman, S. H. (1998). J. Am. Chem. Soc. 120, 90909091. 